\title{
1. Introduction: a new adventure
}

Think about a time when you shared something that you knew that enabled you or your company to do something better or achieve success. What happened? Tell us the story.

Think about a time when one of your colleagues shared something with you that enabled you or your company to do something better or achieve success. What happened? What did you admire in your colleague? Tell us the story.

Take a moment to think about the answers to these questions. Our guess is that you can easily come up with examples of when you felt overburdened, overwhelmed, or stressed at your job, but you may need a few minutes to think about when you appreciated how you work and how valuable your work is to those around you.

This should not be surprising. Traditional applications of organizational change and knowledge sharing rely on finding and solving problems. While this sort of deficit and critical thinking can be valuable in some contexts, it often leaves groups of people feeling frustrated, unsatisfied, and unappreciated.

Appreciative Sharing of Knowledge (ASK) turns this upside down. We developed this model with the idea that it would take the best successes of organizations and attempt to reinforce and build upon them in a positive way while working within the existing culture of the organization. We illustrate the two contrasting approaches to knowledge sharing in Table 1.1 below.

\section{KNOWLEDGE SHARING}

As the 9/11 Commission Report stated, the most identifiable cause of the September 11, 2001 event was the failure among the intelligence agencies to share knowledge. Similar challenges exist in most organizations. Those organizations that have addressed knowledge sharing issues productively are the best in their field. The study of knowledge sharing has tried to replicate practices from the best, but organizations have learned that one size does not fit all. Initially, in the 1990s, practitioners and theorists in the field of knowledge sharing assumed that the reason workers were not able to communicate was because the infrastructure did not exist to help them do 


\section{Table 1.1 Two approaches to knowledge sharing}

\begin{tabular}{ll}
\hline Problem Solving & Appreciative Sharing of Knowledge (ASK) \\
\hline - Knowledge sharing as a & - Knowledge sharing as an \\
problem to be solved & opportunity to be embraced \\
- Identification of problem & - Valuing and appreciating 'what is' \\
- Highlight what is broken & - Affirm what is working \\
- Identify knowledge sharing & - Identify knowledge enablers: \\
problems: What makes people & What makes people share knowledge? \\
hoard knowledge? & - Envision what is possible \\
- Analysis of causes & - Generate future-present scenarios \\
- Generate possible solutions & - Innovating/realizing what will be \\
- Action planning and treatment & - Affirmation as intervention \\
- Fixing as intervention & - Looking at what is present \\
- Looking at what is missing &
\end{tabular}

so. They were convinced that technology would solve the problem. While technology addressed some issues, it fell short in many areas.

With the advent of Lotus Notes and other collaborative software, organizations created, categorized, and sliced information in the hopes that by documenting their information people could share knowledge more effectively. The knowledge sharing toolkit market became saturated with companies that wanted to get on the bandwagon. Corporations spent significant amounts of resources instituting knowledge sharing architectures on the axiom that 'if you build it, they will come'. People who rapidly needed to turn around time sensitive documents like proposals still had to frantically search for current information immediately before the document was due, but the knowledge management tools did not always help because they were not populated with the right information. Often, the tools were not used or contained unusable information and thus became quickly irrelevant, not meeting business needs.

As it became clear that the knowledge management tools were not delivering adequately on their promise, attention was focused on the possibility that the concept of knowledge sharing itself might be an oxymoron. Using the term 'management' implies that knowledge can be planned, organized, and controlled. Since knowledge mostly resides in people's heads, managing it is inherently problematic. The field of knowledge sharing soon made a paradigm shift from knowledge 'management' to knowledge 'sharing'. We began to realize that one of the key reasons that people were not contributing what they knew was because of the perception that they would lose their control on knowledge once it was 


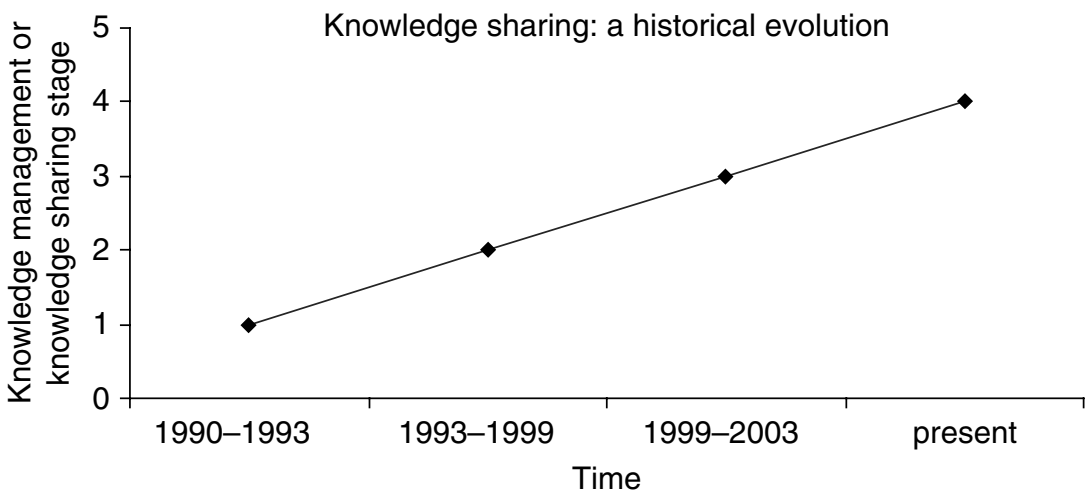

Notes:

- Stage 1 (1990-1993) - Focus on technology infrastructure as the solution to the KM problem

- Stage 2 (1991-1999) - KM tool saturation, widespread use of KM tools, companies 'getting on the bandwagon'

- Stage 3 (1999-2003) - Knowledge sharing as an oxymoron; knowledge cannot be managed but instead must be shared

- Stage 4 (current) - What will help people share knowledge? Appreciative Sharing of Knowledge (ASK).

Figure 1.1 Knowledge sharing: a historical evolution

shared. Subsequently, they might be perceived as less valuable by the company.

Overall, organizations were beginning to understand the power of unleashing knowledge among individuals. What they struggled with was how exactly to unleash that power, given that the very behavior of hoarding the knowledge is what makes employees successful. After all, it is primarily the hyper-competitive culture of many organizations that creates the knowledge hoarding climate. The challenge then is figuring out how to create a knowledge sharing culture.

One of the basic tenets of this book is that if we share knowledge appreciatively, managing knowledge will no longer be an issue. Affirmation may very well be considered a psychological need. Knowledge sharing is one way this need is fulfilled in organizations. If knowledge sharing is done in an appreciative manner, more people are willing to share. The presence of an explicitly appreciative format allows others to say what is on their mind without being questioned, critiqued or put on the defensive.

In this book we expand on the concept of appreciation and show how organizations can create appreciative systems that would institutionalize knowledge sharing and create organizational excellence. We also give 
examples of organizations that have already planted the seeds for the knowledge exchange to happen.

The ASK process can reinvent - in a sustainable manner - the way we think about organizing. By linking practices, artifacts, technologies, and managerial skills, the ASK perspective offers a creative way to manage a wide range of enterprises. As knowledge becomes central to organizations, networks, and markets, the principles and practices of ASK empower a lifeaffirming process of creating value. The first part of this work is focused on providing information in order to re-center ourselves regarding the values of appreciation.

\section{KNOWLEDGE SHARING: A HISTORICAL PERSPECTIVE}

Chapter 2 provides a historical context for the concept of knowledge sharing, part of which is outlined in Figure 1.1. Knowledge sharing is of crucial importance in societal evolution. From our ancestral huntergatherers to current copy machine technicians, knowledge sharing has withstood the test of time. The hunter-gatherers' organizational structure dating back to $10000 \mathrm{BC}$ provides a powerful testimony of knowledge sharing's value. By optimizing and making the best use of the knowledge around them, the hunter-gatherers were able to lead a lifestyle that may be seen as healthy even by today's standards. The hunter-gatherers devoted only a few hours a day to searching for food and sent out only a fraction of the able-bodied foragers each day. By making superior use of their members' knowledge, they were able to survive harsh conditions (Ehin, 2000, p. 58).

Using historical data, we show that the knowledge management methods of hunter-gatherers bear a surprising relevance to contemporary organizational knowledge sharing practices. In this chapter, we also explore other similarities between the rich knowledge sharing cultures rooted in history and those of today's organizations. We conclude by summarizing the lessons learned from the past regarding knowledge sharing and by articulating the concrete ways that learning might apply to the current challenges faced by knowledge sharing. We show that the core element of all successful knowledge cultures of the past was the presence of some form of appreciative system. Such approaches did not define human endeavors in deficit terms but as presence, almost like a form of unconditional acceptance of whatever happened. 


\section{THE GENERATIVE POTENTIAL OF APPRECIATIVE PROCESSES}

In Chapter 3 we explore the concept of appreciation and its generative potential in detail. The concept has strong roots in the philosophical theories of organizational and social sciences. Our goal in this chapter is to establish the foundation for a strong theoretical premise to support the concept of ASK.

The root of appreciation is linked to the Pygmalion and Galatea effects. In Roman mythology, Galatea was the name of a statue of a beautiful woman that was brought to life by Venus, goddess of love, in response to the prayers of the sculptor Pygmalion, who had fallen in love with his creation. Considerable research evidence and literature exist regarding this phenomenon, which is variously called a 'self-fulfilling prophecy', the Pygmalion effect, and the Rosenthal effect (Murphy, Campbell, and Garavan, 1999; Kierein and Gold, 2000; Reynolds, 2002; Rowe and O'Brien, 2002). We have chosen the term 'Galatea effect' to underscore the fact that it is the beauty of the statue that created the desire in Pygmalion. The adoption of the Galatea effect to this aspect of appreciation and knowledge sharing creates significant differences in the way people feel about their capacity to create change in organizations. Essentially, once the knowledge enabling properties are correctly identified, building on them is possible because each individual imagines the ideal future as if it has already happened.

It may seem simple and obvious that people who appreciate each other in the workplace will have a better working relationship than those who have an adversarial relationship. So what then makes it challenging to create an appreciative environment? Over the course of this book we reconcile how to meld our innate appreciative needs with our critical problem solving minds.

The most common misnomer is that appreciation is as simple as 'turning a frown upside down'. However, doing so is not an appreciative act at all. The appreciative approach asks the participants to take a hard look at the reality around them, but affirmatively. But this does not mean ignoring or neglecting the current reality. In this chapter we discuss how the need for the appreciative mindset has historically evolved for knowledge sharing. We provide two ways of looking at knowledge: a retrospective and a prospective approach. In the former, the focus is to look back at what happened with a critical and analytical mindset. This approach, similar to dissection in a biology laboratory, a postmortem of an event, or a case study, has certain merits. It is clearly the dominant approach and has been historically used in a wide range of fields. Examples include the case study 
of a patient in a medical school, the After Action Review that the U.S. Army uses immediately after a training or practice engagement in the field, and the well-known case study approach used in business schools worldwide. The retrospective approach in knowledge sharing looks at what is broken in an organization regarding how knowledge is utilized, isolates the causes for the broken state of affairs, and comes up with remedial actions or 'fixes' to correct the inefficiencies in the system.

In this chapter we also explain the connection between language and knowledge sharing. Encouraged by the strong support expressed in social constructionist writings (Gergen, 1999; Gergen and Thatchenkery, 2004), a specific question is raised in this chapter: what happens when the language to address the organizational knowledge sharing problem itself is changed? What happens if the new approach doesn't even look at problems as problems?

We believe that one such approach that can achieve a significant impact in the knowledge sharing field is Appreciative Sharing of Knowledge. We also show how the ASK approach itself is a derivative of a widely successful organizational development tool called 'appreciative inquiry', originally proposed by Cooperrider and Srivastva in 1987.

\section{HOW TO ASK?}

Chapter 4 explores the process of how to do an ASK initiative. We expect that by the time the reader reaches this stage after reading the case studies of diverse organizations, he or she would have a good sense of the pragmatic issues involved in the process. That is why this chapter provides a systematic way of initiating and completing an ASK initiative for any organization. The chapter is more like a tool kit, or workbook explaining the 'nuts and bolts' of the ASK process, most of which is borrowed from Thatchenkery's previous work titled Appreciative Sharing of Knowledge (Taos Institute, 2005). To give a taste of this approach, we mention a few aspects of the process below, repeating what we have mentioned earlier.

Think about a time when you shared something that you knew that enabled you or your company to do something better or achieve success. What happened? Tell us the story.

Think about a time when one of your colleagues shared something with you that enabled you or your company to do something better or achieve success. What happened? What did you admire in your colleague? Tell us the story.

Such questions provide an outline for the foundation of ASK. Pose them to a group of approximately 30 people and you will be positively impacted 
by the stories that you hear. It is akin to the sparkle that your child feels listening to a teacher speak of Harry Potter-like wizardry.

The reactions to the questions above will help you determine the preexisting climate for knowledge sharing in your organization. If key people view this exercise as a waste of time, that might be your first indication that they are not treating knowledge sharing as a high priority. Senior leaders who might have this attitude may unconsciously encourage knowledge hoarding behavior.

The key ingredient for Appreciative Sharing of Knowledge is climate. Does the organization support sharing? Do people feel they have to hoard their knowledge in order to survive? What has been the history of knowledge sharing initiatives in the organization? Responses to these questions play a key role in deciding when and how you would introduce ASK in an organization.

Many people find organizations to be alienating and oppressive environments. Hence it should not surprise an ASK enthusiast that the appreciative climate is not present in every organization. However, this does not mean that an ASK initiative or approach will not work there. An appreciative climate can be created with top management support. As pointed out in the first chapter, creating an appreciative outlook needs a certain amount of reframing of organizational reality. One has to look consciously to find knowledge sharing events or experiences even when they seem absent at first glance.

\section{Overview of the ASK Process}

As in any organizational change technique, we begin ASK by focusing on the current state or 'what is'. Steps 1, 2, and 3, which are explained in later chapters, will help the practitioner or change agent discover the appreciative temperature of their organization with a series of questions asked in a facilitated session using interviews. The goal will be to capture what has worked so far in the organization and to extract the core processes supporting knowledge sharing. During these steps a set of key themes or 'knowledge enablers' will emerge throughout several of the stories that the participants share. Steps 4, 5, and 6, also described in later chapters, validate the knowledge enablers through a series of interviews and subsequent organizational analyses. Finally, we will also build upon them to create a set of 'future-present scenarios' that are similar to a specific vision of a future that one can perceive in the present. Step 7 takes that list further by expanding and prioritizing them into more manageable and actionable options. The resulting step 8 creates an action plan to make 'what will be' real. Figure 1.2 and Table 1.2 depict and summarize the steps. 


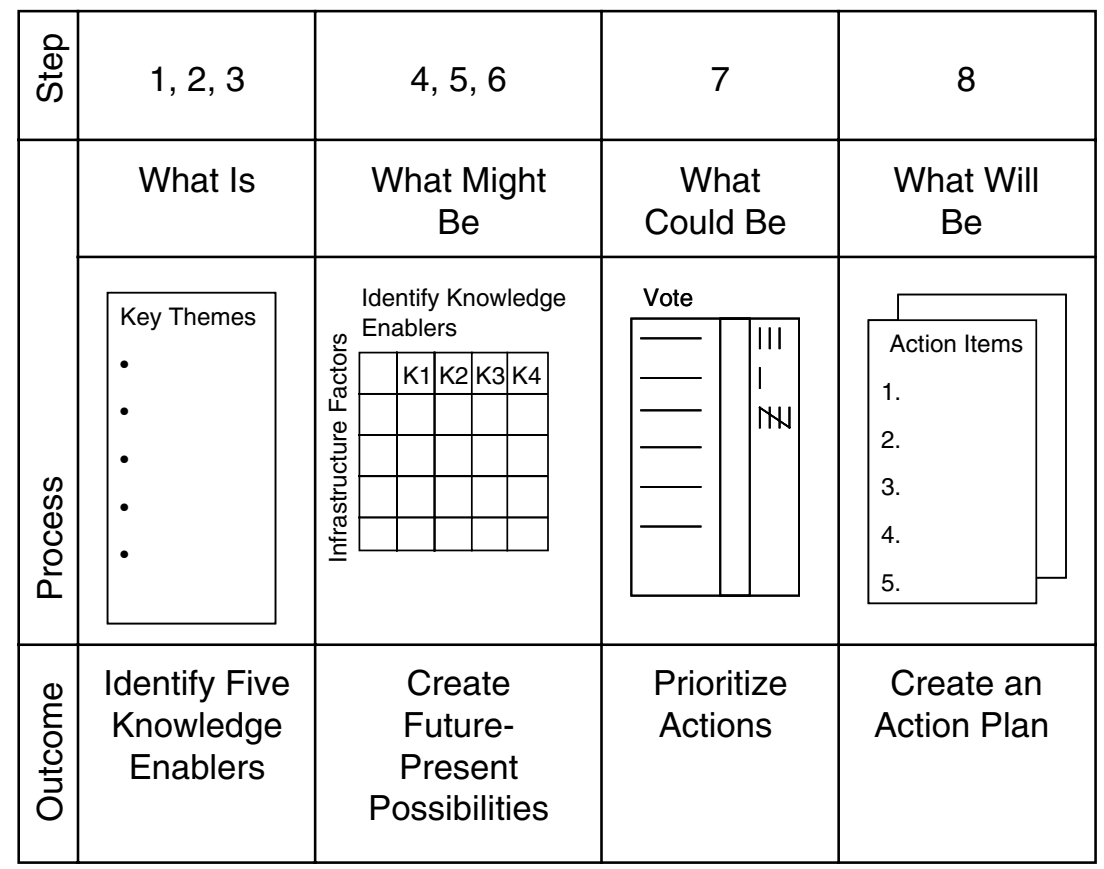

Figure 1.2 Overview of the ASK process

\section{Table 1.2 Overview of the ASK steps}

\begin{tabular}{ll}
\hline Step & Action \\
\hline Step 1 & Negotiating top management commitment and support \\
Step 2 & Presenting the appreciative knowledge sharing paradigm \\
Step 3 & Identification of knowledge enablers \\
Step 4 & Expansion of knowledge enablers using appreciative \\
& interviews designed and conducted by the ASK team \\
Step 5 & Thematic analysis of the data to undertake a knowledge \\
& infrastructure analysis \\
Step 6 & Constructing future-present scenarios \\
Step 7 & Consensual validation of the future-present scenarios \\
Step 8 & Creating and mandating an implementation team \\
\hline
\end{tabular}


These steps can be adopted based on the needs of your organization. By adopting them you may ignite the generative potential that already resides within.

\section{CASE STUDIES}

Chapters 5, 6, and 7 show case studies of five organizations where the ASK approach was used. Each of them starts with a description of the organization and the status of the organization before the ASK process began, and describes how the ASK initiative was negotiated and conducted, lessons learned and future directions. Chapter 5 describes the first of the ASK studies which were conducted in a large financial services organization in the US, the GCB Bank (not the real name), and an environmental information technology company, ITC, which is also a fictional name to protect its identity. Chapter 6 narrates how the process worked in two government organizations: the Maritime Administration (MARAD) and another federal agency, identified only as AFA to protect its identity. Chapter 7 provides an ASK example in a public service organization. These stories give the reader a detailed blueprint for the techniques of designing and implementing an ASK initiative. These chapters also contain an analysis of what factors were present in each of these organizations that helped them become knowledge enabling cultures.

For example, in Chapter 4, ITC wanted to learn more about the knowledge sharing currently taking place. The recently appointed Chief Knowledge Officer wanted to use this to set the foundation for the knowledge management program that she hoped to create. Another objective was to find out 'who knows what' and to learn if ITC's infrastructure could sustain continued growth and support new clients.

With approximately 300 employees ITC was soon going to grow to 500 employees due to increased work demand. Accessing and sharing the knowledge of each and every employee was crucial to its success and further growth. Sharing information, keeping it current, and becoming aware of what other members of the organization were thinking about and doing were necessary to improve the bottom line of the company. ITC was working to increase knowledge sharing - both internally, to ensure that its infrastructure would sustain and encourage growth, and externally, to continually support and increase its number of clients - in order to continue the success of its previous ten years.

The following stories, which we refer to as quintessential stories, appeared in multiple interviews and in a number of other interviews across the team: 
- Gary (fictional name) created a developer's portal for the internet team. He had been an analyst and he started analyzing pages, grouped and linked white papers on new technologies, and built a portal. He started telling people in the company and they'd look at it. And they'd send him stuff to add to it. It has a database you can use to search proposals; it has tools for cutting graphics, web sites are categorized, and it has statistics. What started out as a personal interest became a valuable company tool.

This frequently shared story became a quasi legend within the organization and was used as a testimony that showed employees, current and future, that individual initiatives were recognized and genuinely valued by the company.

- 'My colleague and I were meeting in an ITC conference room, and we could hear through the wall a conversation that a client was having with an ITC consultant. This unintentional eavesdropping became intentional. So, we pulled the consultant out of the room and took him to another conference room. We told him that we heard what he and the client were talking about, and shared our knowledge that was relevant to the client's issues. This informal sharing of knowledge resulted in a good outcome for ITC as well as the client. Our decisions, based on that informal knowledge sharing, were validated by positive feedback from the client.' (Personal interview with author, 2000)

This story enforces the informal organic interactions that help the organization thrive.

- "When the "Green Team" cleaned up a section of highway for the Adopt-a-Highway program, the president provided money to buy plants for the area under the highway sign that let people know ITC adopted this section. What was neat about this project was that it was about giving back to the community. Some people used part of their eight hours of volunteer time for it, and we got to know each other better - those relationships provided a foundation for working with people. Through our informal conversation, we also learned what each other does.' (Personal interview with author, 2000)

These two stories reveal the sense of community and the comfort with knowledge sharing that pervaded ITC. 


\section{SUMMARY, CONCLUSION, AND INVITATIONS}

Chapter 8 provides a summary of what has been provided in the previous seven chapters, generates some conclusions and generalizations on what has been learned, and asks the practitioner community to continue to use the process for organizations of all types. We believe our success with the process has allowed us to share what we have learned about this unique and innovative knowledge sharing tool.

At a time when change is the permanent fixture in organizations' efforts for growth and survival, ASK is a refreshing approach in the field of knowledge sharing. This book shares stories of appreciation and knowledge sharing experienced by several organizations and gives you tips and tools to jump-start a knowledge sharing culture to leave your organization with a culture that realizes its fullest potential. We hope that you will have as much fun engaging with the process as we did. 